\title{
Máscaras y expresión corporal, un vehículo al teatro del alma. Una experiencia de la unidad de rehabilitación del instituto psiquiátrico
}

Carrasco Madariaga, JImena.

\section{- Resumen}

Entre el año 2000 y 2003 se realizó un taller de máscaras y expresión corporal con usuarios de la Unidad de Rehabilitación del Instituto Psiquiátrico José Horwitz Barac. El objetivo inicial era la exploración y desarrollo de las capacidades creativas y expresivas, utilizando técnicas propias de la creatividad y el teatro.

Desde este objetivo inicial, se llegó a un grupo, que fue capaz de llevar a cabo el montaje de una muestra artística, que finalmente se presentó en un festival internacional.

Durante el proceso se observó en cada uno de los integrantes aumento de confianza en las capacidades, desarrollo del sentido de placer y eficacia, aumento de la capacidad de elección, aparición de roles y aumento de la tolerancia a tareas rutinarias.

El presente trabajo sistematiza el proceso del grupo, bajo el modelo teórico de Ocupación Humana de Gary Kielhofner. Para esto se realizaron entrevistas a los profesionales, registros audiovisuales, aplicación de pautas de evaluación individual a los usuarios y registros escritos de las sesiones.

El propósito es dar cuenta en forma sistemática de la progresión en las acciones y consecución de objetivos terapéuticos.

\begin{abstract}
- Abstract
Between the year 2000 and 2003 a masks and corporal expression workshop was performed with users of the Rehabilitation Unit of the Psiquiatric Institute José Horwitz Barac. The initial objective was the exploration and development of the creative and expressive capacities, utilizing techniques belonging to Theatre and creativity.
\end{abstract}

From this initial objective a group was selected, which was able to complete the staging of an artistic piece. This piece was finally presented in an international festival.

During the process of each participant an increase of trust in his/her own capacities was observed, as well as development of the sense of pleasure and efficiency, an increase in the capacity to choose, the appearance of roles, and an increase of tolerance towards everyday tasks.

The following work systemizes the group's process, under the theoretic model of Human Ocupation by Gary Kielhofner. For this work, interviews were performed to the professionals involved, audiovisual records and individual evaluation guides were applied to the users, and written registers of the sessions were taken.

The overall purpose is to give account in a systematic form of the progression in the actions and the obtainment of therapeutic objectives.

Terapeuta Ocupacional. Licenciada en Ciencia de la Ocupación. Docente Carrera de Terapia Ocupacional. Universidad Austral de Chile Contacto > > jimenacarrasco@uach.cl Fono (63) 293510 


\section{Introducción}

Era principios de Abril del año 2003. Después de días de largos ensayos, por fin se acercaba el momento. Los rostros desquiciados, que en un principio me habían parecido tan inexpresivos y lejanos, ahora me transmitían claramente nerviosismo, alegría, confianza y agradecimiento. Escondidos tras el escenario no podíamos ver cuanta gente había, pero por el ruido, deducíamos que era mucha. Finalmente el telón se abrió. Efectivamente el teatro estaba lleno.

Ese día, hicimos lo que habíamos planeado desde hacía más de un año. Les mostramos a todos nuestros sueños, nuestros mundos internos, nuestras fantasías más amadas, todo retratado en "La bella que sueña y el vuelo del pájaro durmiente", nuestro ritual de sanación y nuestro espectáculo para todos quienes lo quisieran ver. Recibimos a cambio, una ovación de aliento y reconocimiento, que sin duda, nunca olvidaremos.

La Unidad de Rehabilitación del Instituto Psiquiátrico tiene como meta la rehabilitación y la reinserción social de personas que han sufrido una enfermedad psiquiátrica severa, y que como consecuencia de ésta, han desarrollado algún grado de discapacidad. Para esto se planifican y llevan a cabo numerosos programas y talleres, donde se estimulan y desarrollan capacidades. Esta unidad está inserta en el Instituto y atiende a tres poblaciones: pacientes crónicos, que no cuentan con apoyo familiar o social y que llevan varios años de permanencia en la institución, pacientes agudos, que se hospitalizan por periodos que no superan los dos años, y finalmente pacientes ambulatorios, cuyas patologías se encuentran estables y no requieren hospitalización pero si de un proceso de rehabilitación y que por esto, asisten durante el día.

Desde su inicio (1996), la Unidad de Rehabilitación ha incorporado la actividad artística como un medio para el logro de sus objetivos. Dentro de estas actividades, en el año 2000 se inició un taller de máscaras y expresión corporal, a cargo de una Terapeuta Ocupacional (Maritza Loyola) y de una Actriz (Elzbieta Mejewska), cuyo objetivo específico era la exploración y el desarrollo de las capacidades creativas y expresivas. El grupo se componía de 12 usuarios. Cuatro de ellos eran ambulatorios y ocho, pertenecían a los diferentes departamentos de Larga Estadía o Crónicos.

El proceso del que se da cuenta en este trabajo, va desde el principio del taller hasta la participación del grupo en el "Festival de Artistas Internados y Externados de hospitales Psiquiatricos", realizado en Mar del Plata Argentina en Abril del 2003.

El marco teórico utilizado para esta sistematización es el Modelo de Ocupación Humana, que define las etapas del proceso de rehabilitación y proporciona indicadores objetivos que permiten determinar cada una de éstas.

El propósito de este trabajo es dar cuenta del proceso terapéutico vivido por los usuarios del taller. Me he planteado como objetivo mostrar la eficacia de la aplicación del Módulo Exploratorio en la intervención con pacientes psiquiátricos con grave discapacidad. También deseo plantear que las diferentes etapas del proceso de Rehabilitación planteadas por el Modelo de Ocupación Humana, son una forma de ordenar y explicar las acciones realizadas en Terapia Ocupacional. Finalmente deseo dejar abierta nuevas interrogantes para futuras investigaciones sobre el uso de técnicas de creatividad y psicodrama en pacientes psiquiátricos y en discapacidad en general.

\section{- Metodología de Recolección de la Información}

Al hacerme cargo del taller, éste ya llevaba un tiempo previo de trabajo, por lo cual fue necesario hacer primero un registro del proceso ya vivido por el grupo. Para esto, la metodología utilizada fue la recopilación de datos sobre las experiencias y el trabajo previo, a través de la realización de 
entrevistas a los usuarios y a la actriz que había estado a cargo del taller desde su inicio. Luego, a partir de la solicitud de los usuarios de preparar una muestra, comencé a registrar el proceso de forma directa. Para esto, apliqué pautas de observación (Cuestionario Volicional) a los usuarios individualmente y elaboré registro escrito de las sesiones.

En resumen para la recolección de los datos, la metodología utilizada fue:

1. Retroactiva:

a.- Aplicación de pauta de evaluación a los usuarios

b.- Aplicación de entrevista semi estructurada a Actriz

\section{Directa:}

a.- Aplicación de Cuestionario volicional en diferentes etapas

b.- Registro escrito y fotográfico de las sesiones

\section{1.a.-Evaluación inicial a los usuarios:}

Al iniciar el trabajo con el grupo, se realizó una evaluación, que tenía por objeto establecer los logros obtenidos en el trabajo previo. Para elaborar una pauta de evaluación se tomó como referencia el Modelo de Ocupación Humana. La pauta de entrevista elaborada, evaluó el nivel de la motivación individual, los factores ambientales físicos y sociales que favorecen o no la participación y el desempeño.

Con el fin de facilitar la aplicación de la entrevista, se propuso que cada uno de los entrevistados, recreara en muñecos a los personajes que había creado a lo largo de los años de trabajo previo. Esto con el apoyo de máscaras y disfraces que se habían utilizado anteriormente y fotografías de los personajes logrados.

Todo los demás compañeros debían colaborar en la recreación de estos personajes y luego podían hacerle preguntas acerca de los mismos. Finalmente yo realizaba la entrevista.

\section{1.b.-Entrevista a Actriz:}

En tres sesiones se llevó a cabo la una entrevista semi estructurada a la actriz Elzbieta Majewska, quien había trabajado con el grupo desde el inicio del taller. El objetivo fue conocer las diferentes etapas del proceso previo. La pauta de entrevista tenía una primera parte que consultaba sobre el proceso de la profesional, una segunda, que consultaba por el proceso del grupo, y una tercera, que preguntaba sobre los procesos individuales de cada integrante.

\section{2.a.- Aplicación de Cuestionario Volicional:}

Se realizó a través de observación directa de cada uno de los integrantes en diferentes etapas: la primera aplicación fue durante la recopilación del material para la elaboración de la muestra. La segunda, durante la elaboración de la estructura dramática de la muestra y la tercera, durante los últimos ensayos previos a la actuación en el festival.

\section{2.b.- Registro escrito y fotográfico:}

Durante cada sesión se tomaban notas referentes a la dinámica grupal, nivel de motivación hacia la actividad, tolerancia del grupo al esfuerzo y a la frustración y proceso creativo. Esto se acompañaba de fotos. 
Una vez obtenido los datos, se identificaron los objetivos planteados y las acciones realizadas. Se establecieron las relaciones entre las acciones y los objetivos y la relación en la secuencia seguida durante el proceso con la planteada por el Modelo de Ocupación Humana.

\section{- Marco Teórico}

\section{EI Modelo de Ocupación Humana ${ }^{(1)}$ :}

Este marco conceptual ve a los seres humanos como un sistema abierto y dinámico, resaltando el comportamiento ocupacional como un proceso organizador.

La Teoría General de los Sistemas plantea que, la actividad espontanea es la característica fundamental de los seres vivos, y que el requerimiento básico para la acción se va volviendo más elaborado a medida que ascendemos por la escala filogenética, llegando en el hombre a conformar la Ocupación.

La acción de los sistemas humanos es necesaria para crear y sustentar su organización, o en otras palabras, la organización en el sistema humano se asienta en la acción. Luego, el Comportamiento Ocupacional es la expresión humana de la acción organizadora, sin embargo, es único para cada persona ya que está dado por la organización interna.

Primero, el comportamiento Ocupacional emana de elecciones que surgen de diferentes motivos. Segundo, el Comportamiento Ocupacional presenta regularidad y patrón. Por último la Ocupación expresa la capacidad subyacente, ya que para su desempeño se pone en juego una amplia gama de habilidades mentales y físicas.

Para explicar de qué manera se elige, organiza y ejecuta el comportamiento Ocupacional, el Modelo de Ocupación Humana conceptualiza al hombre como un sistema compuesto por tres componentes: Volición, habituación y desempeño.

Cada componente es un conjunto organizado e interrelacionado de patrones y procesos que tienen un propósito coherente. De esta forma el propósito del componente volición es seleccionar el comportamiento Ocupacional, mientras que el del componente habituación es determinar la organización del Comportamiento Ocupacional y el del componente de Desempeño es hacer posible la hábil realización de las Ocupaciones. Todos estos componentes poseen su propia organización interna pero se mantienen unidos e interrelacionados dentro de la totalidad mayor del sistema humano en una relación de heterojerarquía. De esta forma, el Comportamiento Ocupacional es visto como simultáneamente influenciado por la aleación de la Volición, la Habituación y el Desempeño, lo que proporciona una explicación sistémica y dinámica del comportamiento Ocupacional.

\section{El proceso de cambio durante la intervención (2):}

Durante la vida, los seres humanos experimentan naturalmente cambios importantes. Comúnmente el cambio ocupacional ocurre a través de una continua progresión desde la exploración al estado de competencia y finalmente estado de logro.

1. La exploración es un periodo de descubrimiento, discriminación de valores, intereses y habilidades. El énfasis para la intervención está puesto en el proceso volicional. 
2. La Competencia es un periodo de aprendizaje de habilidades e interacción con el ambiente. El énfasis para la intervención está puesto en los hábitos, los roles y el desempeño funcional.

3. El Logro es un periodo de desempeño de las habilidades aprendidas en ambientes normales y por lo tanto más desafíenles. El énfasis para la intervención está puesto en la adquisición de roles, la volición el refinamiento de habilidades específicas.

Las personas progresan en estos estados cuando se involucran en nuevos roles, se integran a nuevos ambientes, o cuando organizan sus vidas de acuerdo a circunstancias o eventos importantes o adversos.

El proceso de cambio, durante la intervención utilizando el Modelo de Ocupación Humana, se centra en el cambio de la persona relativo a su desempeño ocupacional. El proceso de remotivación es una estrategia de intervención de Terapia Ocupacional, que plantea módulos, basados en las tres etapas del proceso de cambio ocupacional: Exploración, Competencia y logro.

El proceso de remotivación fue diseñado para promover y facilitar la motivación por la ocupación y está basada en la comprensión de las características y de los desarrollos volicionales únicos de cada persona. De este modo, el proceso de remotivación es complejo e individualizado, por lo que es necesario visualizarlo en diferentes etapas y subetapas que pueden o no ser implementadas simultáneamente y pueden o no ser seguidas en la misma secuencia por todas las personas. Los módulos fueron diseñados para ser guiados por el Terapeuta Ocupacional pero puede ser implementados por una variedad de personas en conjunto con el Terapeuta Ocupacional en ambientes relevantes y significativos para la persona.

La etapa de Exploración es la primera y la más básica en el proceso de cambio. La exploración tiene como objetivo el investigar en un ambiente seguro y relajado los objetos, las actividades, las personas, las habilidades, valores e intereses personales.

La exploración siempre es positiva para el individuo al estar enfocada en el proceso mismo y no en el resultado. Se considera como objetivo principal el descubrimiento y la curiosidad.

Una situación exploratoria debe presentar las siguientes dimensiones:

- Absolución de fracaso: Es la dimensión más importante de esta etapa. Significa interminables oportunidades de experimentar el éxito y el fracaso como formas igualmente aceptadas de participación. Lo importante es el intentar, de modo que la participación jamás es acompañada de consecuencias negativas.

- Dimensión diversión placer: Se define como la curiosidad y placer de descubrir las propias habilidades y "el mundo". La diversión se reafirma por la aceptación del fracaso y por los desafíos y oportunidad que el ambiente otorgue.

- Descubrimiento: Proviene de la curiosidad por el sentido de los objetos, las oportunidades y los otros y de ser validado por la respuesta que se recibe del ambiente físico y social. El descubrimiento debe combinar un ambiente familiar y seguro con uno novedoso y complejo.

- Elección: Se refiere a la libertad dada por el contexto exploratorio. El ambiente es estructurado y provee apoyo y oportunidad de tomar decisiones relacionadas con lo que es importante y valioso para la persona. Permite el desarrollo de sentido de control y competencia, al hacer que la persona se sienta segura, confiado y respetado.

- Ausencia de límites de tiempo: Implica que el proceso de exploración no se limita a una sesión y que por el contrario se extiende a todas las etapas de la intervención. El proceso es individual para cada persona y por lo tanto sólo ésta podrá indicar el momento para pasar a la próxima etapa. 
El módulo exploratorio del proceso de remotivación ha sido desarrollado para facilitar la intervención con personas que sufren severo déficit en su motivación intrínseca. Plantea cuatro etapas con sus respectivos objetivos y acciones: la validación, el desarrollo de la disposición a explorar el ambiente, la facilitación de la elección y el desarrollo de sentido de placer y eficacia.

\section{- Resultados}

A continuación se describe la relación entre los objetivos y las acciones que plantea cada etapa descrita por el Módulo Exploratorio de Modelo de Ocupación Humana ${ }^{(3)}$ y las acciones realizadas efectivamente en el taller. También se mencionan los indicadores volicionales que se observaron en los usuarios en cada una de las etapas. Como es lógico suponer, la aparición de estos indicadores no fue al mismo tiempo, ni con el mismo grado de apoyo en todos los usuarios. Lo importante es señalar que se dieron en todo el grupo.

\section{Etapa de Validación:}

Objetivo: Desarrollo de sentimientos básicos de confianza en el ambiente. Acción: Proveer oportunidades y situaciones significativas en el ambiente Objetivo: Facilitar sentimientos de sentido personal

Acción: Desarrollo de una relación significativa

En esta primera etapa, el trabajo personal del profesional a cargo, es la base del trabajo con el usuario. En el caso de la actriz, también fue así. A fin de brindar un ambiente que diera confianza a los usuarios, es decir, que no presentara para ellos mayores demandas, fue necesario primero, un trabajo personal de la actriz, quien nunca había trabajado con este tipo de pacientes. Este trabajo consistió en confrontar los propios prejuicios y temores, además de las propias expectativas, de la profesional a cargo, en relación al trabajo con ellos. La profesional debió confrontarse con las incapacidades de los usuarios, espacialmente las expresivas. Sólo así se pudo llegar a valorar las potencialidades y a las personas que habían en el grupo. Esto permitió que desde el principio se creara un ambiente acogedor y sin demandas. Por otra parte, para el desarrollo de una relación significativa, la profesional relata, que debió realizar un trabajo de validación de los mundos internos de cada uno, incluyendo los aspectos que eran incomprensibles para ella. Esto era fundamental ya que estos mundos internos serían la base del trabajo creativo.

Los indicadores volicionales logrados en esta etapa fueron:

- Los usuarios mostraron curiosidad por la actividad

- Los usuarios mostraron que la actividad era significativa.

\section{Etapa de Desarrollo de Disposición Para Explorar el Ambiente:}

Objetivos: Desarrollar sentimientos exploratorios.

Acción: Facilitar la exploración de nuevos ambientes, alternativas y personas.

Objetivo: Reafirmar sentimientos de sentido personal.

Acción: Apoyar la exploración de nuevas alternativas.

Objetivo: Reafirmar sentimientos de confianza en el ambiente.

Acción: Continuar la provisión de oportunidades y situaciones significativas en el ambiente.

Durante esta etapa, se realizó la exploración de nuevos ambientes y alternativas que servirían de base para el proceso creativo. Se hizo un trabajo de exploración del propio cuerpo, a través de ejercicios: dibujos, trabajos con el espejo y con otros compañeros. 
También se realizó un trabajo de exploración de los mundos internos. Para esto último se facilitaron materiales que apoyaban la representación, tales como máscaras y disfraces. Todo esto siempre en el contexto de un proceso de exploración, donde el ambiente fue definido como lúdico y sin demandas de ningún tipo. Los materiales no eran entregados directamente a cada uno, sino que estaban a disposición para quien los quisiera utilizar y sólo se hacía la invitación a manipularlos. Luego, cada acción realizada era reconocida primero por la profesional y luego, por todo el grupo. Había aplausos, palabras de agradecimiento y elogios.

Los indicadores Volicionales logrados en esta etapa fueron:

- Los usuarios iniciaron acciones tales como manipular máscaras, bailar y dialogar. - Los usuarios Intentaros cosas nuevas tales como disfrazarse y elaborar diálogos.

\section{Etapa de Facilitación de la Elección:}

Objetivo: Facilitar proceso de auto validación por encuentro de valores e intereses en la actividad. Acción: Proveer oportunidad de elección y facilitar la expresión de valores e intereses, apoyando la elección

Objetivo: Reafirmar el sentido de si mismo y confianza personal

Acción Apoyar participación en acciones que la persona elige

A medida que los usuarios fueron explorando sus propios cuerpos, sus mundos internos y el ambiente, fueron surgiendo personajes que se apoderaban de cada uno de ellos.

Algunos primeros, y otros después, todos comenzaron a preferir determinadas máscaras y vestuarios hasta llegar a conformar personajes, con características bien definidas. Las preferencias de cada uno eran apoyadas y estimuladas a través de aplausos y comentarios. Se brindaba el espacio para la aparición y expresión de personajes cada vez que se solicitaba y se estimulaba al resto del grupo para que lo apoyara. Una vez establecidos los personajes de cada uno (podían ser más de uno) comenzaron a aparecer temáticas y relaciones entre éstos, lo que también fue apoyado de la misma forma que en el caso de la aparición de los personajes. En este punto, cabe señalar, que tanto los personajes como las historias que aparecieron tenían directa relación con las características personales y las historias de vida de los usuarios. Dado que no es el objetivo de este trabajo hacer un análisis de los procesos creativos y proyectivos que se dieron, no se ahondará en la descripción de cada personaje ni de las historias, pero si es importante destacar cómo los usuarios se fueron encontrando con aspectos personales, y cómo el permitirles la elección de éstos aspectos, facilitó el proceso de auto validación.

Los Indicadores Volicionales logrados en esta etapa fueron:

- Los usuarios indicaron preferencia por determinados personajes y tramas.

- Los usuarios permanecieron involucrados en la actividad.

\section{Etapa de Desarrollo de Sentido de Placer y Eficacia:}

Objetivo: Desarrollo de confianza personal y auto validación

Acción: Participación en roles significativos y toma de decisiones

Objetivo: Desarrollo de conocimiento de propias capacidades

Acción: Relación de colaboración con otros, con aumento progresivo del desafío

Desde los personajes y las tramas que se fueron creando, comenzaron a surgir nuevas necesidades que fueron percibidas por el grupo y que dieron origen a relaciones de colaboración y finalmente roles diferenciados. Había que realizar acciones que eran necesarias para el desarrollo del trabajo creativo. Algunos comenzaron a asumir en forma permanente las tareas de orden de la 
sala, estructuración del espacio (escenario y público), guardando y cuidado de los materiales, reunión del grupo, etc. En un caso en particular, un usuario asumió en forma espontánea el rol de libretista, siendo quien creaba los textos.

Es en esta etapa, en forma espontánea surge la necesidad de mostrar el trabajo realizado, y en consecuencia, la propuesta de preparar una muestra. Esta petición, sin duda, respondía al desarrollo de Confianza personal y Conocimiento las Propias Capacidades en los usuarios. Se había hecho necesario que Participaran de la toma de decisiones en relación al trabajo del grupo, y que se diera una nueva etapa con aumento progresivo del desafío.

Los Indicadores Volicionales logrados en esta etapa fueron:

- Los usuarios intentaron resolver problemas prácticos para la realización de la actividad. - Los usuarios indicaron como objetivo la realización de una muestra.

\section{Etapa de Adquisición de Competencia:}

Una vez que los usuarios deciden realizar una muestra, se hace necesario redefinir el objetivo de la actividad.

Para la realización de un montaje, se debía trabajar en función de obtener un producto final. Una puesta en escena, con una estructura, requería de un trabajo que demandaba continuidad en el tiempo y perseverancia a los usuarios.

Se debía generar una nueva instancia, que tuviera continuidad con el taller de máscaras y expresión corporal, pero con características diferentes.

Fue entonces necesario, implementar un nuevo programa en el cual el objetivo ya no era sólo la exploración de las capacidades expresivas y creativas, sino, en el que estas capacidades ya exploradas, se desarrollaran y se utilizaran en un proceso creativo que finalmente diera un producto concreto. Este producto sería un espectáculo teatral.

El espectáculo fue planteado de modo de ser presentado en cualquier instancia y no sólo dentro de nuestra institución, a fin de dar un abordaje más normalizado a la iniciativa.

De este modo se cambió el nombre del programa a "Teatro del Alma" y se determinaron nuevos objetivos:

- Brindar una instancia de validación de los logros obtenidos en el taller de máscaras y expresión corporal.

- Desarrollar capacidades creativas y expresivas.

Al comenzar esta nueva etapa se realizó una revisión de los resultados obtenidos en el taller de máscaras y expresión corporal. Cada uno seleccionó los personajes y las historias que consideró habían sido sus mayores logros.

A partir de este material se comenzó a estructurar un espacio o ambiente y una trama. En este proceso se siguieron definiendo los personajes y las tramas hasta llegar a una historia hilada que contenía a todos los personajes, y al menos elementos de los mundos imaginarios de cada uno de los usuarios. 
En un principio se probaron diferentes alternativas sin aún definir un libreto ni una secuencia, hasta que fueron los propios usuarios quienes solicitaron a la actriz que definiera una estructura y comenzar a trabajar sobre ésta.

Se accedió a esta solicitud y por primera vez la profesional asumió activamente la dirección de la muestra. En este momento se hace evidente el cambio de orientación en los objetivos de la actividad, lo que es percibido y asumido por los usuarios; desde el objetivo de auto complacencia se pasa al objetivo de crear un efecto en otros, y el grupo está dispuesto a asumir una dirección y una limitación en las acciones en la medida en que esto tiene sentido para el logro del nuevo objetivo.

Una vez definida la estructura de la muestra, se comienza con los ensayos, que requerían de trabajo repetitivo y de cooperación entre los integrantes. Con esto, se reafirmaron los roles que habían aparecido en las etapas anteriores y surgieron nuevos.

Cuando por fin se logró un producto a la altura de un espectáculo, se realizaron las presentaciones. La primera se llevó a cabo en el acto central del aniversario 150 del Instituto Psiquiátrico el día 8 de agosto de 2002. Luego vinieron otras presentaciones a familiares y amigos y a la comunidad, como la realizada en el ex hospital San José y actual centro cultural. Después de cada presentación se discutían las posibles modificaciones y se volvía a trabajar en la exploración de las capacidades creativas y expresivas a fin de buscar nuevo material para incorporar a la muestra. En este momento, el espectáculo no tenía nombre. Sólo a principios del año 2002 decidimos como grupo, qué era lo central de lo que queríamos transmitir al público y surgió el nombre de la obra: "La Bella que Sueña y el Vuelo del Pájaro Durmiente". Este nombre hace referencia a los dos personajes que quisimos poner en el centro de la trama: uno, la Bella, hace alusión a alguien que fue maldecida a dormir para siempre, sin embargo en este mundo de sueños, da vida a todos los demás. El otro, el Pájaro Durmiente, hace alusión a un ser que también vive durmiendo pero que a veces se despierta, y cuando lo logra, vuela.

Como ya se ha mencionado, el objetivo de este trabajo no es al análisis de los elementos creativos que surgieron, sino del proceso vivido en términos de comportamiento ocupacional. Por esto, no ahondaré en este punto, pero si deseo señalar, que nuevamente cobró importancia, el que los elementos trabajados tuvieran directa relación con las historias de vida de cada uno de ellos. Esto hacía aún más significativa la actividad.

En Diciembre del año 2002, llega la invitación de la red Argentina de Arte y Salud Mental para participar del $7^{\circ}$ Festival de Artistas Internados y Externados de Hospitales Psiquiátricos a realizarse en Abril del año 2003 en Mar del Plata Argentina. El grupo toma la invitación como un nuevo desafío y se propone concluir el trabajo realizado con la presentación en dicho festival.

Finalmente y luego un arduo trabajo el grupo se presentó en el Teatro Pueyrreidón, (ubicado en el centro de la ciudad de Mar del Plata) lleno de espectadores. La presentación fue llevada a cabo de forma impecable y el público respondió con aplausos y ovación.

Al volver al Hotel, los integrantes de las otras delegaciones volvieron a aplaudir al grupo en el casino a la hora de la cena.

\section{Logros Alcanzados:}

Al concluir el trabajo, se pudo determinar que los principales logros obtenidos, en relación al comportamiento ocupacional de los usuarios, fueron:

- Desarrollo de las capacidades Creativas y Expresivas de los Usuarios

- Aumento de la Confianza Personal de los Usuarios 
- Desarrollo del Sentido de logro en los usuarios

- Desarrollo de relaciones significativas y de cooperación entre los usuarios

- Desarrollo de Intención comunicativa en los usuarios.

Con relación a las tareas realizadas, se observó un avance en las cinco dimensiones que plantea el Modelo de Ocupación Humana:

- Complejidad: De simples juegos se llegó al montaje de un espectáculo.

- Límites temporales: de acciones breves y sin límites temporales se llegó a la realización de ensayos de hasta dos horas de duración.

- Reglas: desde una libertad en las elección de las acciones a realizar y en el grado de participación, se pasó a una actividad que requería cumplir acciones específicas y establecidas y participación activa de todos los integrantes

- Seriedad / Diversión: de una actividad que en principio provocaba sentido de placer por la libre exploración de las propias habilidades y del ambiente y que era considerada "divertida", se pasó a una actividad con sentido productivo, es decir que contenía un sentido social, además del sentido de placer y eficacia que los usuarios desarrollaron.

- Dimensión social: de una actividad de carácter privado, que no requería de mayor interacción con otros, se pasó a una actividad de carácter social que consistía en una muestra del trabajo realizado a un grupo de espectadores.

\section{Conclusiones y Discusión}

Quisiera, citar lo referido por la actriz Elzbieta Majewska durante una de las entrevistas: "Terapia Ocupacional es el proceso desde que alguien tiene las ganas de hacer algo, hasta que de alguna manera lo logra. Puede ser cualquier cosa, un mueble, una obra de teatro, lo importante es que sea aquello que la persona soñó consciente o inconscientemente en poder hacer. Cuando esa persona lo logra, y alguien la acompañó y creyó en ella, ese alguien hizo Terapia Ocupacional".

Cito esta expresión porque creo que encierra, en sentido amplio, la idea central de lo que fue el trabajo con estos usuarios. Teniendo en cuenta que, el quehacer de la Terapia Ocupacional no es fácilmente reconocible para quienes no se relacionan directamente con él, me parece importante rescatar la impresión de alguien que participó en un proceso de rehabilitación y trabajó mano a mano con un Terapeuta Ocupacional.

En primer lugar, deseo plantear la importancia de la aplicación sistemática del módulo exploratorio en pacientes con graves alteraciones en su comportamiento Ocupacional. Considero que este trabajo previo facilita el que las personas puedan encontrarse con sus reales motivaciones. Sólo a partir de éstas se pueden obtener logros que sean significativos para nuestros usuarios.

Planteo también que el respeto por las motivaciones individuales, responde a la capacidad que tenga el profesional de ver a cada persona y su individualidad. Esto es producto de una forma de enfrentar a la persona con discapacidad, que tiene que ver con aspectos personales del profesional y que va más allá de cualquier modelo teórico.

Luego, el Modelo de Ocupación Humana es una herramienta teórico-práctica con la cual contamos los Terapeutas Ocupacionales y que se basa en los fundamentos filosóficos y éticos de nuestra profesión. Además permite una comprensión de los procesos que determinan la motivación de las personas hacia la ocupación, con lo cual nos entrega herramientas para trabajar en base a ésta. 
El Modelo de Ocupación Humana, nos permite explicar y ordenar nuestra intervención en cualquiera de las áreas en que nos desempeñemos. De esta forma, podemos utilizar herramientas y técnicas de múltiples áreas y disciplinas que tienen que ver con el comportamiento ocupacional de nuestros usuarios, como lo fue en este caso, la creatividad y el teatro. Esto, sin dejar de lado este Marco Teórico que es propio y que nos ordena la forma de utilización de otras técnicas.

Finalmente, quisiera dejar abierta la inquietud para futuros trabajos sobre la utilización de técnicas de desarrollo de creatividad y psicodrama en personas que sufren de una discapacidad. Esto es importante si tenemos en cuenta que hoy en día, el arte es una actividad que está siendo utilizada en el trabajo con diferentes tipos de discapacidades y con buenos resultados. Creo que como Terapeutas Ocupacionales, tenemos mucho que decir en la justificación, sistematización, validación y promoción de este tipo de actividades como herramienta terapéutica.

\section{Agradecimientos:}

Agradezco al Instituto Psiquiátrico Dr. José Horwitz Barak, por haber apoyado esta experiencia, especialmente a su director Dr. Ignacio Morlans, al Jefe de Servicio de Rehabilitación, Dr. Carlos Cid, y a la Jefa de la Unidad de Rehabilitación, Terapeuta Ocupacional Maritza Loyola. También agradezco a mi amiga y colaboradora, la Actriz Elzbieta Majewska por su dedicación y su ayuda incondicional, y a los usuarios que hicieron posible este trabajo.

\section{Referencias}

(1) DE LAS HERAS CARMEN GLORIA (2004) "Teoría y aplicación del Modelo de Ocupación Humana" Capítulo "Modelo de Ocupación Humana de Gary Kielhofner, edición 1995". Editado por Reencuentros Chile).

(2) DE LAS HERAS CARMEN GLORIA (2004) "Teoría y aplicación del Modelo de Ocupación Humana" Capítulos "Estados de cambio", "Principios de intervención", "Resumen proceso de remotivación". Editado por Reencuentros Chile.

(3) DE LAS HERAS CARMEN GLORIA (1999) Modulo Exploratorio. Editado por Reencuentros Chile. 